\title{
Streptomyces roseoverticillatus produces two different poly(amino acid)s: lariat-shaped $\gamma$-poly(L-glutamic acid) and $\varepsilon$-poly(L-lysine)
}

\author{
Masanobu Nishikawa and Kei Kobayashi
}

Correspondence

Masanobu Nishikawa

mnishikawa@bio-ribs.com

Received 31 March 2009

Revised 9 June 2009

Accepted 15 June 2009

\author{
Research Institute for Biological Sciences Okayama (RIBS Okayama), 7549-1 Yoshikawa, \\ Kibichuo-cho, Kaga-gun, Okayama 716-1241, Japan
}

The poly(amino acid)s $\gamma$-poly(DL-glutamic acid) (gPGA) and $\varepsilon$-poly(L-lysine) (ePL) are known to be natural linear poly(amino acid)s secreted by Bacillus spp. and Streptomyces spp., respectively. In this study, a Streptomyces strain producing both ePL and gPGA was identified. Mass spectrometry and other analyses revealed that the gPGA is a mixture of oligomers consisting of 10-13 L-glutamic acid residues linked by isopeptide bonds. In contrast to the known Bacillus gPGA, the glutamic acid oligomers have a cyclodehydrated structure in each molecule. We previously reported that the ePL molecules secreted by the same Streptomyces strain disperse only slightly in an agar culture plate, as though they were larger molecules. This phenomenon is explicable by the observed polyion complex formation between the glutamic acid oligomers and ePLs. The glutamic acid oligomers control the ePL's dispersion, which would also affect the spatial distribution of the ePL's antimicrobial activity. Therefore, gene clustering or common use of the gene was presumed for biosynthesis of the two poly(amino acid)s. However, no gene for biosynthesis of the glutamic acid oligomer was found in the neighbouring region of that for ePL biosynthesis, and the glutamic acid oligomer was produced by a mutant in which the ePL biosynthetic gene was inactivated by gene disruption.

\section{INTRODUCTION}

$\gamma$-Poly(DL-glutamic acid) (gPGA), and $\varepsilon$-poly(L-lysine) (ePL) are naturally occurring linear poly(amino acid)s (Shima \& Sakai, 1981a; Ashiuchi \& Misono, 2002; Saimura et al., 2008). The former exhibits characteristics of large molecules $(10-1000 \mathrm{kDa})$ but the latter is small $(1-5 \mathrm{kDa})$. Both are constructed by isopeptide bonds: $\alpha$-amino- $\gamma$ carboxyl bonds in gPGA and $\varepsilon$-amino- $\alpha$-carboxyl bonds in ePL. Each is produced enzymically, by either an ADPforming process or an AMP-forming process during ATP consumption (Ashiuchi et al., 2001; Kawai et al., 2003; Yamanaka et al., 2008). It has been shown that gPGA is secreted by Bacillus subtilis and related species and that ePL is secreted by Streptomyces and related species (see references above). To our knowledge, no report has described a bacterial group producing another poly(amino acid) simultaneously. The differences in the biosynthetic

Abbreviations: ePL, $\varepsilon$-poly-(L-lysine); gPGA, $\gamma$-poly(DL-glutamic acid); IsgPGA, lariat-shaped $\gamma$-poly(L-glutamic acid); NAC, $N$-acetyl-L-cysteine; OPA, o-phthalaldehyde.

The GenBank/EMBL/DDBJ accession number for the $7267 \mathrm{bp}$ nucleotide sequence containing the ePL biosynthetic gene of $S$. roseoverticillatus is AB477240.

Two supplementary figures are available with the online version of this paper. process and the separated distribution of such poly (amino acid)-producing species show no correlation of biosynthesis between the two poly(amino acid)s.

We previously isolated a bacterium, Streptomyces roseoverticillatus MN-10, which produced ePL with a unique characteristic (Nishikawa \& Ogawa, 2002): although the average molecular size was about $3 \mathrm{kDa}$, the ePL only slightly dispersed in an agar plate, in contrast to ePL secreted by other strains $(2-5 \mathrm{kDa})$. A correlation exists between molecular dispersion in an agar plate and molecular mass. However, the ePLs produced by strain $\mathrm{MN}-10$ deviate from the correlation: they behave as though the molecules are larger than the real molecular mass. The degree of molecular dispersion in an agar plate can usually be explained by chemical interactions such as the electrostatic force and hydrogen bonds of ePL molecules with other molecules, including counter-ions, water and polysaccharides in agar gel. We verified that no chemical modification occurs in the MN-10-produced ePL. Therefore, we presumed the existence of anions that were able to repress the polycationic feature of ePL. In this paper, we report unusual oligomers consisting of glutamic acid, which can interact with ePL.

Recently, Yamanaka et al. (2008) opened the door to progressive studies of ePL biosynthesis by cloning the 
relevant gene, pls. The question arose whether biosynthesis of the glutamic acid oligomers correlates with that of ePL. In this study, we isolated the pls gene from S. roseoverticillatus $\mathrm{MN}-10$, determined the gene organization around this gene, disrupted the MN-10 pls, and examined the effect of the disruption on the biosynthesis of the glutamic acid oligomers.

\section{METHODS}

Strain and culture condition. Streptomyces roseoverticillatus strain MN-10 (Nishikawa \& Ogawa, 2002) was used. Spores grown on ISP4 agar (Difco) were inoculated into a $200 \mathrm{ml}$ Erlenmeyer shaking flask containing $40 \mathrm{ml}$ of a semi-synthetic culture medium according to Shima \& Sakai (1981b). In some experiments, ${ }^{15} \mathrm{~N}$-labelled ammonium sulfate was used instead of $\left({ }^{14} \mathrm{NH}_{4}\right)_{2} \mathrm{SO}_{4}$ and ${ }^{13} \mathrm{C}$-labelled Dglucose was used instead of glycerol. Culturing was conducted at $28{ }^{\circ} \mathrm{C}$ for $2-5$ days.

Poly(amino acid) extraction. Each culture filtrate was applied to a cartridge filled with ion-exchange resin. SepPak QMA (quaternary ammonium moiety; Waters) and SepPak CM (carboxymethyl group) were used for anion exchange and cation exchange, respectively. After washing the cartridges with $0.2 \mathrm{M}$ acetic acid, effluents obtained by pouring through $0.1 \mathrm{M} \mathrm{HCl}$ were collected and dried in vacuum.

Mass spectrometry. Crude samples containing poly(amino acid)s were analysed by MALDI-TOF/MS with an Autoflex 2 mass spectrometer (Bruker Daltonics). As a matrix, 2,5-dihydroxybenzoic acid was used.

Poly(amino acid) purification. The anionic poly(amino acid) extracted from the culture filtrate was subjected to HPLC. After acidification to neutralize the electrical charge, the anionic poly (amino acid) was injected to a liquid chromatograph (LaChrom L5000 series; Hitachi High-Technologies) equipped with a C18 reverse-phase-type column $(4.6 \times 150 \mathrm{~mm}$, Hydrosphere C18; YMC), pre-equilibrated with $0.1 \mathrm{M} \mathrm{HCl}$. By increasing the concentration of acetonitrile in the mobile phase, anionic poly(amino acid) was eluted selectively, with monitoring of UV absorbance (200 nm).

Residual monomer determination (GC/MS). The purified anionic poly(amino acid) was hydrolysed in the presence of $6 \mathrm{M} \mathrm{HCl}$ at $105{ }^{\circ} \mathrm{C}$ for $10 \mathrm{~h}$. The acid hydrolysate was dried in vacuum, and was chemically derivatized with $\mathrm{N}$-(tert-butyldimethylsilyl)- $\mathrm{N}$-methyltrifluoroacetamide (MTBSTFA) (Kelly \& Taylor, 1976). The sample was injected into a GC/MS device (Clarus 500 MS; Perkin Elmer) equipped with a nonpolar-type column (DB-5 MS; Agilent Technologies).

Residual monomer determination (HPLC). The acid hydrolysate described above was derivatized chemically using $o$-phthalaldehyde (OPA) and $\mathrm{N}$-acetyl-L-cysteine (NAC) (Nimura \& Kinoshita, 1986); the amino acid was separated using HPLC with a Hydrosphere C18 column, pre-equilibrated with $0.1 \mathrm{M}$ sodium acetate ( $\mathrm{pH}$ 6). Fluorescent signals (excitation, $350 \mathrm{~nm}$; emission, $450 \mathrm{~nm}$ ) were recorded while the concentration of methanol was increasing in the mobile phase.

Peptidase-sensitivity assay. Purified anionic poly(amino acid) (30 pmol) was exposed to V8 protease (0.01 unit, endoproteinase Glu-C from Staphylococcus aureus; Sigma-Aldrich) in $0.2 \mathrm{M}$ aqueous ammonium hydrogen carbonate solution $(\mathrm{pH} 8)$ at $37^{\circ} \mathrm{C}$ for $30 \mathrm{~min}$. The enzymic activity was verified using a synthetic substrate, $\mathrm{N}-\mathrm{t}$ BOC-L-glutamic acid. Similarly, the same poly(amino acid)
(60 pmol) was exposed to carboxypeptidase G (0.007 unit, from Pseudomonas sp.; Sigma-Aldrich) in $0.2 \mathrm{M}$ Tris/HCl buffer ( $\mathrm{pH} 7.5$ ) containing $1 \mathrm{mM}$ zinc chloride at $30{ }^{\circ} \mathrm{C}$ for $20 \mathrm{~min}$. After enzyme treatments followed by derivatization with OPA/NAC, samples were subjected to HPLC similarly to the poly(amino acid) purification described above.

Reconstruction of polyion complex. FITC-labelled ePL was prepared by conjugating about $1-3$ fluorophor groups to the amino groups of ePL ranging from 3.5 to $4.5 \mathrm{kDa}$. The purified anionic poly (amino acid) (15 pmol) was mixed with the FITC-labelled ePL $(15 \mathrm{pmol})$ in $5 \mathrm{mM}$ potassium phosphate buffer $(\mathrm{pH} 7)$ at $28{ }^{\circ} \mathrm{C}$ for $20 \mathrm{~min}$. The sample was injected into the HPLC device equipped with a gel-filtration column $(7.8 \times 300 \mathrm{~mm}$, TSK-G2000SWXL; Toso $)$ equilibrated with the same buffer. Fluorescent signals (excitation, $495 \mathrm{~nm}$; emission, $520 \mathrm{~nm}$ ) were recorded with isocratic flow at $0.5 \mathrm{ml} \mathrm{min}{ }^{-1}$.

Gene cloning and gene disruption. An internal region within the ePL biosynthetic gene of $S$. roseoverticillatus strain $\mathrm{MN}-10$ was amplified using PCR with primers $5^{\prime}$-cgacgcctcctgcgargaratgtg- $3^{\prime}$ and $5^{\prime}$-cttggcgcccagcarncknarcca- $3^{\prime}$. These two PCR primers respectively match the conserved regions FDASCEEMW and WLRLLGAKVGK, which were found among Streptomyces albulus Pls (AB385841) and homologues, e.g. Streptomyces avermitilis SAV6633 (AB070954) (see Supplementary Fig. S2). Subsequently, based on the DNA sequence, genomic DNA walking was conducted using the inverse PCR technique. Finally, a 7267 bp nucleotide sequence containing the ePL biosynthetic gene was determined (DDBJ accession no. AB477240). An approximately $4.8 \mathrm{kbp}$ PCR fragment containing the ePL synthetase gene was amplified using primers $5^{\prime}$-caagaattcagagatacccatggcgtcag- $3^{\prime}$ and $5^{\prime}$-cgcgaattcggtgacggtgaaggtgtacg- $3^{\prime}$, and was inserted into an EcoRI site situated in the lac $Z^{\prime}$ of a pUC18-based vector, pMN-9. pMN-9 had the origin of transfer (oriT) from pPM803 (Mazodier et al., 1989) and a modified spectinomycin (Spc) resistance gene (aadA) from pHP45omega (Prentki \& Krisch, 1984) with a mutation in the 170th codon (TTA to CTC) instead of an ampicillin resistance gene. To produce a plasmid for disrupting the chromosomal ePL synthetase gene, the apramycin (Apra) resistance gene $(\operatorname{aac}(3) I V)$ was inserted into a NruI site situated near the central region of the MN-10 ePL-synthetase. Through intergeneric conjugation, the resultant plasmid was introduced into $\mathrm{MN}-10$ from Escherichia coli ET12567 carrying pUZ8002. Transconjugants were first selected using $\operatorname{Apra}^{\mathrm{R}}$. Subsequently, candidates for the gene disruptant were selected using $\mathrm{Spc}^{\mathrm{R}}$. The $\mathrm{Apra}^{\mathrm{R}} / \mathrm{Spc}^{\mathrm{S}}$ clones were subjected to PCR analysis to verify the correct gene disruption.

\section{RESULTS}

\section{Determination of the primary structure of the acidic substance}

S. roseoverticillatus strain $\mathrm{MN}-10$ secreted an unknown acidic substance into the culture broth. A sample eluted from the anion-exchange resin with $0.1 \mathrm{M} \mathrm{HCl}$ yielded a set of signal peaks with $\mathrm{m} / \mathrm{z}$ range of 1000-2000 (Fig. 1a). The arrayed peaks at an equal interval of 129.1 showed the presence of a repeating structure composed of units with a molecular mass of 129.1. MS/MS analysis supported this idea (Fig. 1b). A precursor ion with $\mathrm{m} / z 1548.5$ generated a set of fragmented ions arrayed at an equal interval of 129.1. Therefore, the substance is a homopolymer. The value obtained by subtracting 18 (water) from the molecular 
(a)

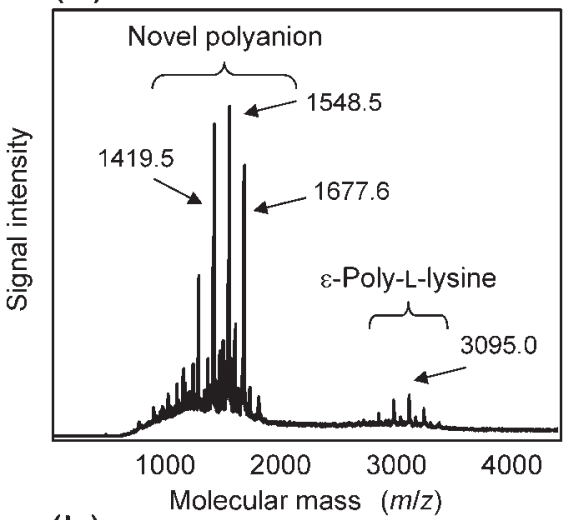

(b)

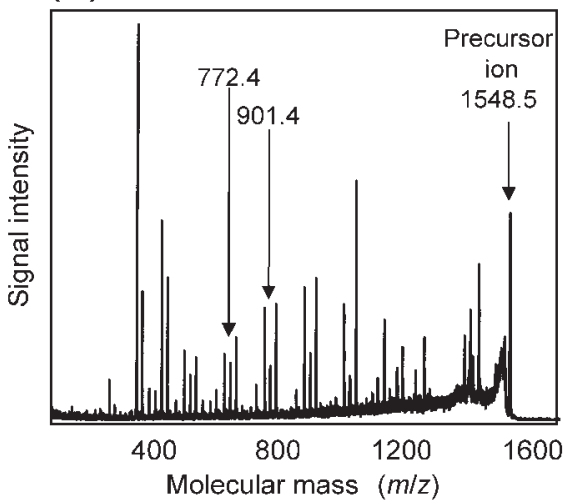

(c)

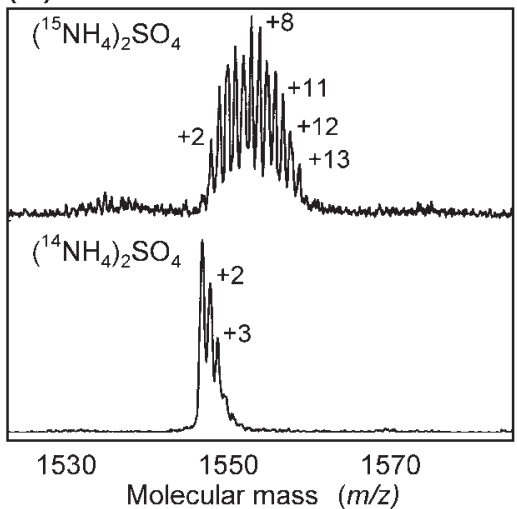

(d)

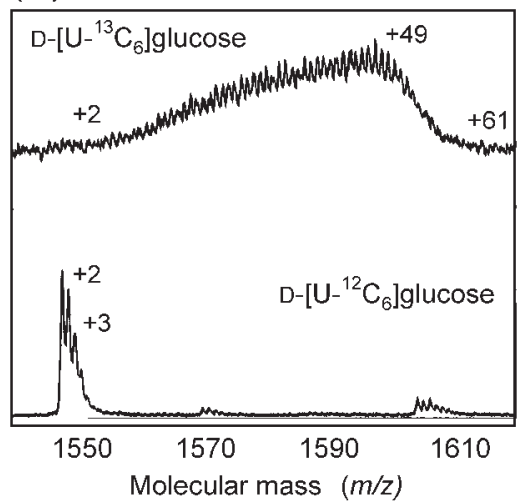

Fig. 1. Positive-ion MALDI-TOF mass spectra of the poly(glutamic acid) secreted into the culture broth by $S$. roseoverticillatus $\mathrm{MN}-10$. An effluent fraction from the anion-exchange resin was subjected to MS (a) and MS/MS (b). The $\mathrm{m} / \mathrm{z}$ values of several peaks are shown; ePLs were detected as well as poly(glutamic acid)s. Similarly, the poly(glutamic acid) secreted by $\mathrm{MN}-10$ during the culture in the presence of stable isotopes was analysed. The culture medium contained either $\left({ }^{15} \mathrm{NH}_{4}\right)_{2} \mathrm{SO}_{4}$ (c) or $\mathrm{D}-\left[\mathrm{U}-{ }^{13} \mathrm{C}_{6}\right]$ glucose (d). Control experiments, in the absence of stable isotopes, are shown below each spectrum. Numbers following ' + ' indicate the mass increment from monoisotopic mass. mass (e.g. 1548.5) must be divisible by 129.1 (the repeating unit) without a remainder if the polymer is a linear molecule produced by a dehydration reaction similar to other biosynthetic polymers such as gPGA and ePL. However, it is not divisible [(1548.5-1-18)/ $129.1=11.85]$. Although it is difficult to conclude that a signal of $\mathrm{m} / z 1548.5$ originates from an $[\mathrm{M}+\mathrm{H}]^{+}$ion, assuming that this is true, 1548.5 is divisible almost to 12 by 129.1 without subtracting 18 [(1548.5-1)/129.1=11.99], which indicates that the polymer contains a circular structure by dehydration in each molecule. Namely, a signal of $\mathrm{m} / z 1548.5$ corresponds to a $12 \mathrm{mer}$ that contains a circular structure. The molecular mass distribution is narrow and judged to be nearly monodisperse.

To estimate the number of nitrogen atoms and carbon atoms in a repeating unit, stable nitrogen and carbon isotopes were added to the culture. The results (Fig. 1c, d) imply that the 12 mer contains $12-13$ nitrogen atoms and about 60 carbon atoms maximally per molecule. Therefore, the numbers of nitrogen atoms and carbon atoms per repeating unit are calculated as 1 and 5, respectively. The molecular mass (129.1 Da) and the atomic composition $\left(\mathrm{N}_{1}, \mathrm{C}_{5}\right)$ indicated that the repeating unit might be glutamic acid.

To identify the repeating unit, the acidic polymer produced by MN-10 was hydrolysed to monomers with $\mathrm{HCl}$. GC/MS analysis of the silylated monomer determined that the repeating unit is glutamic acid (Fig. 2a). Enantioseparation using HPLC showed that almost all the glutamic acids are of the L-configuration (Fig. 2b): the L/D ratio was determined to be 98.3/1.7. A signal corresponding to $\mathrm{L}^{-}$ aspartic acid was also observed in the same sample (Fig. 2b), but no polymer containing L-aspartic acid was detected (data not shown). The L-aspartic acid was thought to be an impurity (see Supplementary Fig. S1). As described above, the L-glutamic acid oligomer bound to an anion-exchange resin. Furthermore, it was not released from the resin with $0.2 \mathrm{M}$ acetic acid ( $\mathrm{pH}$ 2.7) but was released with $0.1 \mathrm{M} \mathrm{HCl}(\mathrm{pH} \mathrm{1.0)}$. Considering that the $\mathrm{p} K_{\mathrm{a}}$ values of L-glutamic acid are 2.19 ( $\alpha$-carboxyl group) and 4.25 ( $\gamma$-carboxyl group), it is most probable that almost all the $\alpha$-carboxyl groups are free and that the $\gamma$ carboxyl group of a glutamic acid residue forms an isopeptide bond with the $\alpha$-amino group of the next one.

The putative isopeptide bond was examined using peptidases acting on L-glutamic acid residues. The V8 protease is an endopeptidase cleaving the peptide bond between the $\alpha$-carboxyl group of a glutamic acid residue and the $\alpha$-amino group of the neighbouring amino acid residue, including glutamic acid, which occurs in general proteins. The MN-10 L-glutamic acid oligomer was resistant to V8 protease (Fig. 3a). Carboxypeptidase G is an exopeptidase cleaving the isopeptide bond between the 
(a)

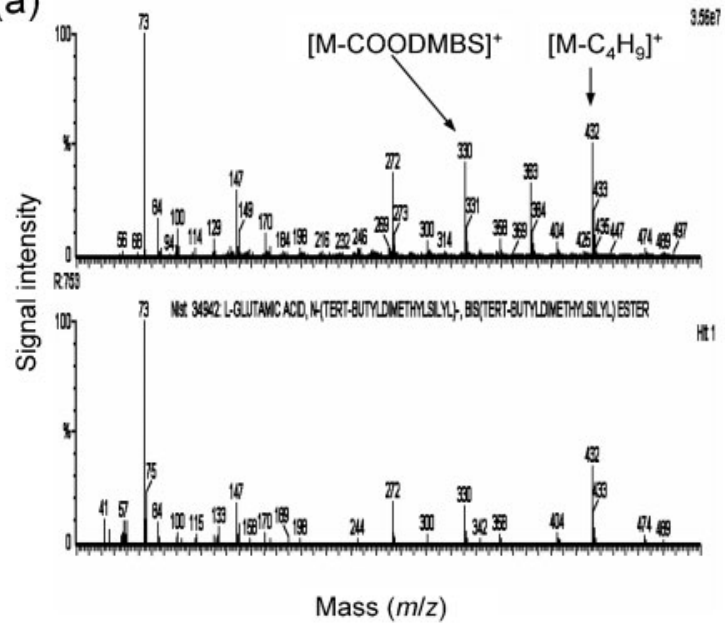

(b)

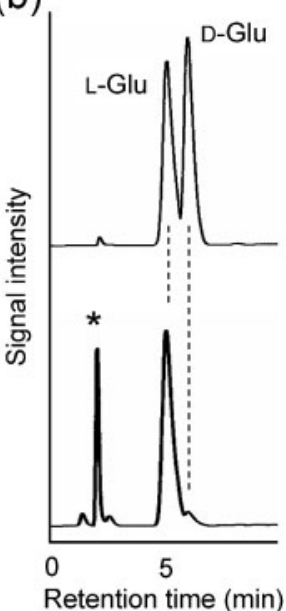

Fig. 2. Positive-ion GC mass spectrum (a) and HPLC chromatogram (b) of acid hydrolysate of the poly(glutamic acid) produced by S. roseoverticillatus MN-10. (a) The intramolecular dissociation pattern of a peak having the same GC retention time as authentic Lglutamic acid is shown (upper spectrum) with that of L-glutamic acid, which the automatic NIST library search program selected as the most probable candidate (lower spectrum). (b) Enantioseparations of the same hydrolysate (lower chromatogram) and authentic standards, L-glutamic acid and D-glutamic acid (upper chromatogram) are shown. L-Aspartic acid was also detected in the hydrolysate (asterisk). $\gamma$-carboxyl group of an L-glutamic acid residue and the $\alpha$ amino group of the next L-glutamic acid residue, which occurs in polyglutamyl folic acid and Bacillus poly(glutamic acid). The MN-10 poly(L-glutamic acid) was sensitive to carboxypeptidase $\mathrm{G}$ (Fig. 3b). These results indicate that the putative isopeptide bonds exist. The amount of released L-glutamic monomer is nearly equal to the total amount of L-glutamic acid composing the oligomer. Therefore, the oligomer was almost completely hydrolysed by carboxypeptidase G. Taking these results together with resistance to Edman degradation and resistance to derivatization using OPA (data not shown), we conclude that the oligomer produced by $\mathrm{MN}-10$ is a $\gamma$ poly(L-glutamic acid) consisting of 10-13 residues with a circular structure around the $\mathrm{N}$-terminus. The oligomer is inferred not to be a complete circular molecule but instead to be a lariat-shaped molecule with a free $\gamma$-carboxyl group (a)

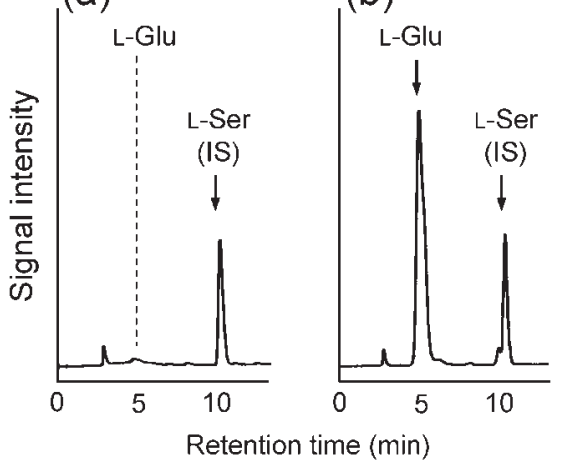

Fig. 3. HPLC chromatograms showing action of peptidases towards the poly(glutamic acid) produced by $S$. roseoverticillatus $\mathrm{MN}-10$. The poly(glutamic acid) was exposed to either V8 protease (a) or carboxypeptidase $\mathrm{G}$ (b); the resultant samples were derivatized with OPC/NAC and analysed using HPLC.
(Fig. 4). Hereinafter, the novel substance is designated lsgPGA [(lariat-shaped gamma-poly(L-glutamic acid)].

\section{Polyion complex formation}

MN-10 was isolated as an ePL-producing bacterium. Although MN-10 secreted ls-gPGA, ePL also accumulated in the culture supernatant. In theory, because ePL is polycationic, it should not bind to anion-exchange resin. However, the sample eluted from anion-exchange resin contained ePL together with 1s-gPGA (Fig. 1a). Polyion complexes seemed to form between ePL and ls-gPGA in the culture broth. To examine formation of such a polyion complex using molecular mass measurement, FITClabelled ePL and purified 1s-gPGA were mixed at a $1: 1$ ratio in $5 \mathrm{mM}$ phosphate buffer $(\mathrm{pH} 7)$, then subjected to size-exclusion HPLC. Formation of the polyion complex was verified as the appearance of an additional signal peak originating from the substance larger than the molecular mass of the free FITC-labelled ePL (Fig. 5).

\section{Correlation of the biosynthesis of $\mathrm{ePL}$ and Is-gPGA}

To examine the correlation between biosynthesis of ePL and that of 1s-gPGA, the MN-10 ePL synthetase gene was isolated using PCR with degenerate primers. The genomic DNA fragment obtained contained a gene encoding a protein of 1339 amino acid residues. The amino acid sequence is homologous overall to that of Pls, an ePL synthetase from Streptomyces albulus NBRC14147 (Yamanaka et al., 2008): identity, $71.5 \%$; similarity, $85.5 \%$. There is no significant difference such as notable segment addition or segment deletion between the two enzyme proteins (see Supplementary Fig. S2). Therefore, the gene was concluded to be a pls homologue in MN-10. The substrate amino acid that was predicted to be adenylated is not L-glutamic acid but L-lysine (Supplementary Fig. S2). The MN-10 pls gene is located 


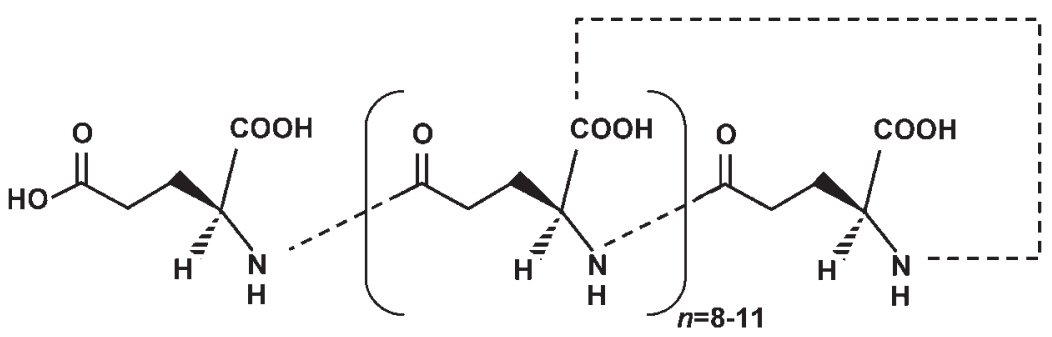

Fig. 4. Most probable chemical structure of lariat-shaped $\gamma$-poly(L-glutamic acid) (IsgPGA) produced by $S$. roseoverticillatus $\mathrm{MN}$ 10.

between a gene encoding a putative $\mathrm{ABC}$ transporter homologue of Streptomyces hygroscopicus ABC42565 (DQ314862) and S. avermitilis SAV1670 (BA000030) and a gene encoding a putative difB (defective in fruiting) homologue of Streptomyces sp. DS570461 (B4VF97) and Stigmatella aurantiaca AAMD01000160 (Q08SN6). No gene encoding probable ls-gPGA synthesis enzymes exists in the neighbouring region of the pls gene.

The wild MN-10 chromosomal pls gene was disrupted by exchange with pls:: Apra $^{\mathrm{R}}$ (Fig. 6a) to examine whether $p l s$ controls the structure of ls-gPGA. The culture filtrate of the gene disruptant was divided into two portions, which were subjected to solid-phase extraction using either a cation exchanger or an anion exchanger. The samples were analysed using MALDI-TOF/MS. The gene disruptant had lost the ability to produce ePL as expected (Fig. 6b). No influence of the pls disruption was found on the ability to produce ls-gPGA (Fig. 6c): thus synthesis of ls-gPGA is independent of pls.

\section{DISCUSSION}

Although the final structural determination requires further experimentation, the acidic substance secreted by S. roseoverticillatus $\mathrm{MN}-10$ is a lariat-shaped oligomer

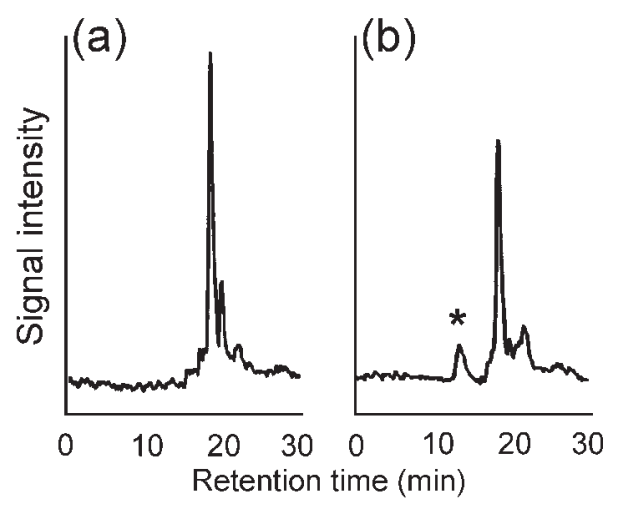

Fig. 5. HPLC chromatogram showing formation of the polyion complex between the poly(glutamic acid) produced by $S$. roseoverticillatus $\mathrm{MN}-10$ and FITC-labelled ePL: (a) FITC-ePL, (b) FITC-ePL plus poly(glutamic acid). The asterisk denotes the polyion complex formed. composed of 10-13 L-glutamic acid residues linked via isopeptide bonds (ls-gPGA). To our knowledge, occurrence of ls-gPGA has never been reported previously. Isopeptides consisting of glutamic acid residues are widely distributed among Bacillus spp. The molecular mass of the Bacillus poly(glutamic acid) is $10-1000 \mathrm{kDa}$, considerably larger than that of ls-gPGA. The Bacillus polymer comprises DLglutamic acid residues. As another example, L-glutamic acid oligomers serve in the chemical modification of folic acids. As described above, ls-gPGA has no components other than L-glutamic acid. Aside from these two unrelated poly(glutamic acid)s, no report of a similar substance appears to exist in the literature.

Our experiments indicate that the L-glutamic acid oligomer has no free amino group. The $\mathrm{N}$-terminus is blocked by undetermined but probable cyclodehydration. Positive-ion MALDI-TOF/MS uses a soft ionization method and mainly detects $[\mathrm{M}+\mathrm{H}]^{+}$and $[\mathrm{M}+2 \mathrm{H}]^{2+}$ ions under normal conditions. It remains unclear whether our observed ion (Fig. 1a) was $[\mathrm{M}+\mathrm{H}]^{+}$because, apparently, no group to be protonated exists in ls-gPGA. Intramolecular dissociation is known to be induced by MALDI when excessive energy beyond that required for desorption-ionization is applied. We were cautious about using laser power to avoid inducing intramolecular dissociation, but if our observed ions (Fig. 1a) were such fragmented ions, our observation, which supported the existence of the cyclodehydrated structure, would weaken the evidence. However, considering that we detected no substances other than L-glutamic acid and Laspartic acid after acid hydrolysis (Fig. 2b), and that we detected negative ions with nearly the same molecular mass by negative-ion MALDI-MS (data not shown), there is little possibility for intramolecular dissociation from poly(glutamic acid) or other peptides. Therefore, we conclude that lsgPGA is the most probable structure.

The ls-gPGA is an oppositely charged partner forming polyion complexes with ePL. Our observation - that ePL with molecular mass ranging from 2 to $4 \mathrm{kDa}$ only slightly disperses in an agar plate, as if it were a larger molecule - is explicable by the existence of such polyion complexes. In nature, some Streptomyces spp. probably secrete ePL to inhibit other microbes. The simultaneous production of lsgPGA and ePL seems to decrease the dispersion of ePL and to reduce the antimicrobial activity of ePL due to its polycationic nature. However, the biological importance of the polyion complex remains unexplained. Although ls- 


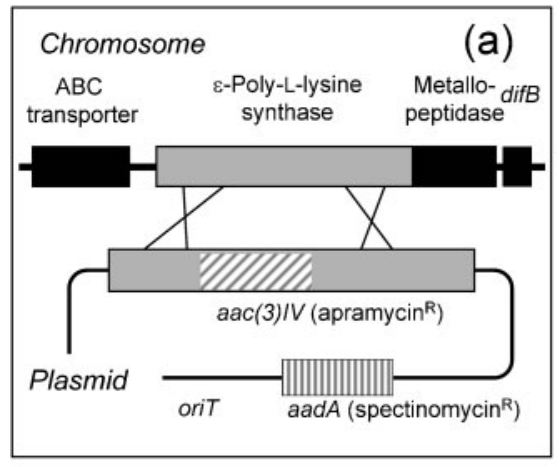

gPGA and ePL mutually interact, no relation exists between the two polymers with regard to common use of synthesizing enzymes or gene clustering of the relevant genes. In future work we will investigate the antimicrobial action of the ePLls-gPGA complex to clarify its biological significance.

\section{ACKNOWLEDGEMENTS}

We are grateful to Chisso Corp. (Japan), Inbiotec (Spain), the John Innes Centre (UK) and the National Institute for Genetics (Japan) for providing us with useful experimental materials.

\section{REFERENCES}

Ashiuchi, M. \& Misono, H. (2002). Biochemistry and molecular genetics of poly $\gamma$-glutamate synthesis. Appl Microbiol Biotechnol 59, 9-14.

Ashiuchi, M., Nawa, C., Kamei, T., Song, J. J., Hong, S. P., Sung, M. H., Soda, K. \& Misono, H. (2001). Physiological and biochemical characteristics of poly gamma-glutamate synthetase complex of Bacillus subtilis. Eur J Biochem 268, 5321-5328.

Kawai, T., Kubota, T., Hiraki, J. \& Izumi, Y. (2003). Biosynthesis of epsilon-poly L-lysine in a cell-free system of Streptomyces albulus. Biochem Biophys Res Commun 311, 635-640.

Kelly, R. W. \& Taylor, P. L. (1976). tert-Butyl dimethylsilyl ethers as derivatives for qualitative analysis of steroids and prostaglandins by gas phase methods. Anal Chem 48, 465-467.
Mazodier, P., Petter, R. \& Thompson, C. (1989). Intergeneric conjugation between Escherichia coli and Streptomyces species. J Bacteriol 171, 3583-3585.

Nimura, N. \& Kinoshita, T. (1986). $o$-Phthalaldehyde- $N$-acetyl cysteine as a chiral derivatization reagent for liquid chromatographic optical resolution of amino acid enantiomers and its application to conventional amino acid analysis. J Chromatogr A 352, 160-177.

Nishikawa, M. \& Ogawa, K. (2002). Distribution of microbes producing antimicrobial epsilon-poly L-lysine polymers in soil microflora determined by using a novel method. Appl Environ Microbiol 68, 3575-3581.

Prentki, P. \& Krisch, H. M. (1984). In vitro insertional mutagenesis with a selectable DNA fragment. Gene 29, 303-313.

Saimura, M., Takehara, M., Mizukami, S., Kataoka, K. \& Hirohara, H. (2008). Biosynthesis of nearly monodispersed poly(epsilon-L-lysine) in Streptomyces species. Biotechnol Lett 30, 377-385.

Shima, S. \& Sakai, H. (1981a). Poly L-lysine produced by Streptomyces. Part II. Taxonomy and fermentation studies. Agric Biol Chem 45, 2497-2502.

Shima, S. \& Sakai, H. (1981b). Poly L-lysine produced by Streptomyces. Part III. Chemical studies. Agric Biol Chem 45, 25032508.

Yamanaka, K., Maruyama, C., Takagi, H. \& Hamano, Y. (2008). Epsilon-Poly L-lysine dispersity is controlled using a highly unusual nonribosomal peptide synthetase. Nat Chem Biol 4, 766-772.

Edited by: Jung-Hye Roe 